\title{
Wave Propagation in the Magnetic Network on the Sun
}

\author{
S. S. Hasan ${ }^{1}$, G. Vigeesh ${ }^{1}$ and A. A. van Ballegooijen ${ }^{2}$ \\ ${ }^{1}$ Indian Institute of Astrophysics, Bangalore, India, \\ email: hasan@iiap.res.in \\ ${ }^{2}$ Harvard-Smithsonian Centre for Astrophysics, Cambridge MA, U.S.A
}

\begin{abstract}
Hasan et al. (2005) have recently presented 2-D dynamical calculations on wave propagation in in the magnetic network of the Sun. The latter is idealized as consisting of nonpotential flux tubes in the quiet solar chromosphere. It is of interest to understand how the nature of wave propagation is influenced by the choice of initial equilibrium configuration of the magnetic field. We examine this by comparing the earlier calculations with those when the network is modelled as a potential structure. Our calculations demonstrate that the nature of the wave propagation is significantly different, particularly the transport of energy which for the potential case, occurs more isotropically than for the non-potential configuration.
\end{abstract}

Keywords. Sun: magnetic fields - magnetohydrodynamics (MHD) - Sun: photosphere - oscillations

It is well known that the magnetic network in the solar atmosphere consists of intense magnetic field elements, in which MHD waves are likely to play an important role in their dynamics and energy transport. Recent studies by Hasan et. al. (2005, hereafter Paper I) have examined the wave propagation in a network element due to periodic motions at its base. These elements or flux tubes are modelled as non-potential structures in which the field drops sharply at the interface with the ambient medium. In the present work, we examine an alternative model of the network to investigate how sensitive the dynamics is to the form of the initial configuration.

Let us first consider a flux tube in the magnetic network having a non-potential field structure. The structure of the flux tube at the initial instant is assumed to be in static equilibrium and is determined by the solving the magnetostatic force balance equation (for details see Paper I). The resultant field configuration has a sharp interface across which the field drops rapidly though continuously to a small value. This region with a very small magnetic field (of the order of a few Gauss) is the ambient medium.

We examine wave excitation in this structure due to a periodic transverse motions of the lower boundary, with a period of $24 \mathrm{~s}$ and an amplitude of $750 \mathrm{~m} \mathrm{~s}$. The time dependent evolution of these configurations is followed using a numerical method described by Steiner et al. (1994), that uses the flux corrected transport algorithm. From Fig. 1(a) (as well as Paper I), the footpoint motions of the flux tube are an efficient source of acoustic waves at the tube-ambient medium interface. In the ambient medium they are visible as a semicircular pattern that propagates outwards at the local sound speed. Close to the tube axis, the pattern is dominated by slow longitudinal acoustic-like waves with large temperature fluctuations. As expected, the flow is essentially along the field lines.

We now turn to the case of a potential field configuration for the network, similar to the one studied by Rosenthal et al. (2002) and Bogdan et al. (2003). The field structure for 

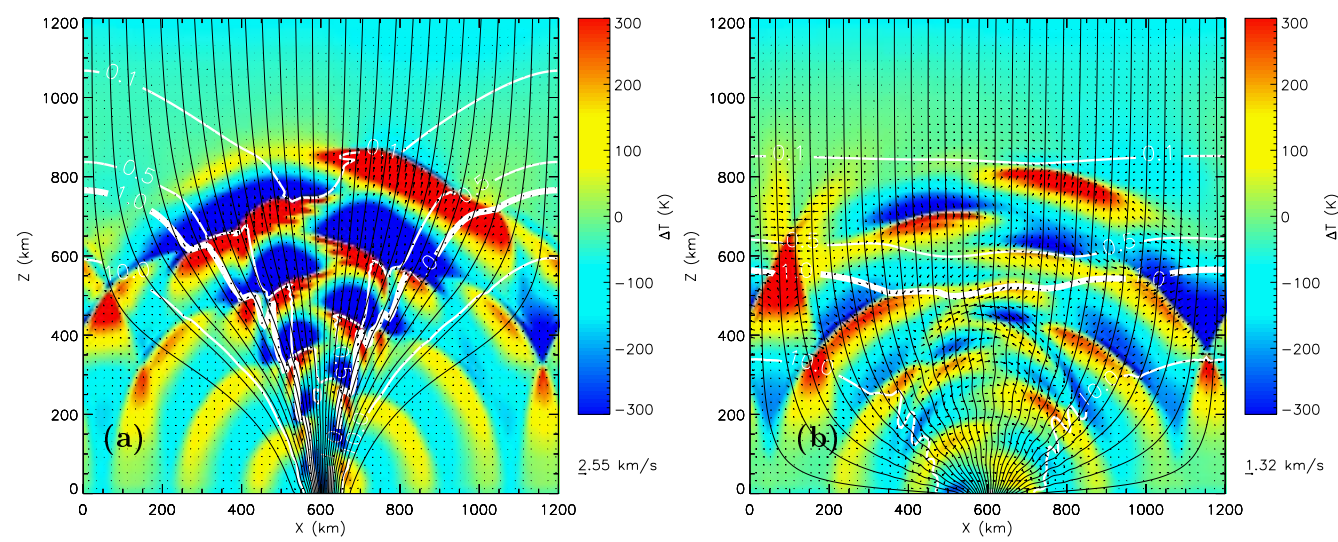

Figure 1. Flow pattern(arrows) and temperature perturbation(colors) at $110 \mathrm{~s}$ a configuration with (a) a non-potential field and (b) potential field due to a periodic horizontal motion at the lower boundary with an amplitude of $750 \mathrm{~m} \mathrm{~s}^{-1}$ and a period of $24 \mathrm{~s}$. The thin black curves represent the magnetic field lines and the heavy white curves contours of constant $\beta$ corresponding to $\beta=0.1,0.5,1.0$ (thick curve), and 10 .

the magnetic field is constructed in terms of a scalar function that satisfies the Laplace equation with boundary conditions at the base $(y=0)$, identical to those for the nonpotential (flux tube) case. The field strength and $\beta$ on the axis at the base are $1400 \mathrm{G}$ and 0.5 respectively (identical to the flux tube case). It should be noted that the magnetic field above the base is more uniformly spread in the horizontal direction and there is no sharp interface that separates the field from the ambient medium.

Using the same periodic excitation as before, we find from Fig. 1(b), that below the surface corresponding to $\beta=1$, the wave pattern generated by horizontal displacements at the flux tube base consists essentially of slow modes (Alfvénic in character), where the motions are transverse to the field and produce distortions in the field lines. The motions at the lower boundary also produce compressions and rarefactions $\left(180^{\circ}\right.$ out of phase on opposite sides of the axis) that generate fast modes (acoustic-like) waves that propagate ahead of the slow wave front. Above the $\beta=1$ layer, where the Alfvén speed is significantly higher than the sound speed, we see a longitudinal slow wave that is acoustic in nature, propagating at the acoustic speed.

In summary, we find that the nature of wave propagation is different for the flux tube and potential field configurations. In the latter, the lower part of the atmosphere (where $\beta>1$ ), has both Alfvénic (transverse) and acoustic waves travelling almost isotropically, whereas in the former, the pattern in the central regions of the flux tube is dominated by acoustic waves that produce larger temperature fluctuations, stronger shocks and consequently greater heating in the higher layers of the atmosphere. Thus, flux tubes appear to be promising in transporting energy to the upper chromosphere.

\section{References}

Hasan, S. S., van Ballegooiijen, A.A., Kalkofen \& W., Steiner, O. 2005, ApJ. 631, 1270

Bogdan, T. J. et al. 2003, ApJ 599, 626

Hasan, S. S., Kalkofen, W., van Ballegoiijen, A. A., Ulmschneider, P. 2003, ApJ 585, 1138

Rosenthal, C.S. et al. 2002, ApJ 564, 508

Steiner, O., Knölker M., \& Schüssler, M. 1994, NATO ASI Ser. C-433, (Dordrecht: Kluwer), p. 441 